\title{
Socially-Mediated Negotiation for Obstacle Avoidance in Collective Transport
}

\author{
Eliseo Ferrante, Manuele Brambilla, Mauro Birattari, and Marco Dorigo
}

\begin{abstract}
In this paper, we present a novel method for performing collective transport in the presence of obstacles. Three robots are physically connected to an object to be transported from a start to a goal location. The task is particularly challenging because the robots have a heterogeneous perception of the environment. In fact, the goal and the obstacles can be perceived only by some of the robots. Hence, the task requires appropriate negotiation of the direction among the robots. We developed a novel negotiation strategy in order to tackle this challenge. We perform experiments in simulation. In the experiments, we analyze efficiency in an environment with only one obstacle, and robustness in an environment with several obstacles.
\end{abstract}

\section{Introduction}

The ability of robots to move in a coordinated fashion is of central importance for the multi-robot research community. Research in coordinated motion can be divided in two categories. In the first category we find works in multi-robot formation, were no physical connection between robots is assumed. In the second category, we find works in collective transport and coordinated motion where there is a physical connection between the robots or between the robots and the object to be transported.

Works in multi-robot formation have been documented in some surveys [1, 2], where the authors compare centralized vs. decentralized approaches. The most studied decentralized method in this area are social potentials [3] and artificial physics [4].

In collective transport, a group of robots has to cooperate in order to transport an object that, because of its weight, cannot be transported by a single robot. The task we are interested in is particularly challenging because communication between the

Eliseo Ferrante $\cdot$ Manuele Brambilla $\cdot$ Mauro Birattari $\cdot$ Marco Dorigo IRIDIA, CoDE, Université Libre de Bruxelles, Brussels, Belgium e-mail: \{eferrant, mbrambil, mbiro, mdorigo\} @ulb.ac.be 
robots is only local, robots have no access to global information and they coordinate using a decentralized approach.

Several works on collective transport were developed using centralized approaches like leader-following behaviors. In these works [5, 6, 7], a group of robots is able to collectively push/pull an object. In order to coordinate their movements, the robots follow a leader that has the knowledge of the goal area or of the path.

Balch [8] was one of the first to study the impact of communication in multi-robot systems. Later, Donald et al. [9] and Yamada et al. [10], studied collective transport with limited communication. In the first work [9], robots had to transport an object without a goal location, whereas in the second work [10] robots had to carry an heavy object towards a common goal determined by a light emitter (photo-taxis).

Campo et al. [11] investigated the use of goal negotiation strategies for performing collective transport to a given goal location. The robots used by the authors had only a noisy perception of the goal, or they were not able to perceive the goal at all. Furthermore, each of the robots used LEDs and an on-board camera to perceive the orientation of the other robots, and used this information to compute an average direction of motion.

Groß and Dorigo [12] used artificial evolution to synthesize a neural network to achieve collective transport. Their robots were able to cope with objects of different size and weight as well as with groups of different size (from 4 to 16). The authors were able to obtain three different transport strategies. In the first one, the robots directly connect to the object and pull it. In the second one, the robots connect to each other (self-assembly) and to the object in order to pull it. In the third strategy, the robots create a physical loop around the object. This last strategy involves a high number of robots and a small (but heavy) object.

Trianni et al. [13] studied a task similar to obstacle avoidance in collective transport. They call it collective hole-avoidance. In their task, robots are physically connected to each other, and they have to navigate in an environment with holes. The authors used artificial evolution for the synthesis of robots' neural network controllers, and studied different communication strategies among the robots: no direct communication, handcrafted signaling and communication induced by artificial evolution. Differently from the work described in this paper, in Trianni et al. [13] no object had to be transported. Furthermore, the robots did not have a specific goal direction on where to go but they were rather exploring the environment while avoiding holes.

Baldassarre et al. [14] studied a task similar to the one studied by in Trianni et al. [13]. In their study, physically connected robots collectively navigate in an environment with obstacles, furrows and holes and a light source to be found. The authors used artificial evolution to synthetize a behavior able to integrate these three sub-behaviors in a coherent fashion: collective motion, collective obstacle avoidance and collective light approaching. However, the synthetized behavior heavily exploited the traction sensor, a specialized sensor that is able to detect forces exerted by the connected robots and that might not be available on all robotics platforms.

In this paper, a group of three simulated robots have to transport an object from a start to a goal location in an environment with obstacles. Almost all tasks studied so far in the literature consider collective transport in an obstacle-free environment 


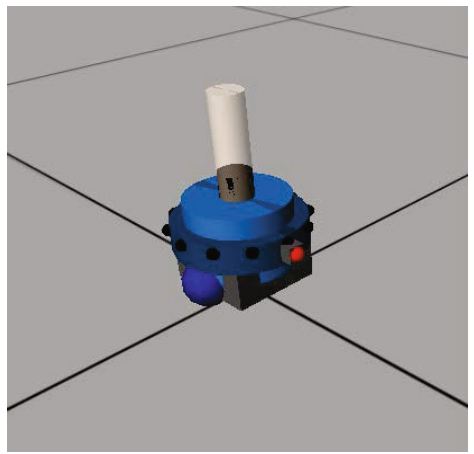

(a)

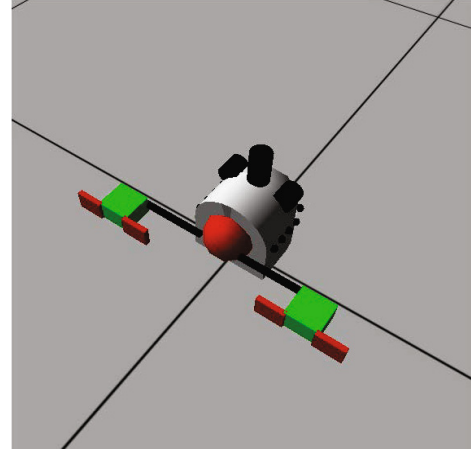

(b)

Fig. 1 Picture of the simulated foot-bot (a) and of the hand-bot, which is the irregularly shaped object to be carried (b)

where a goal location is given, with two notable exceptions. In Trianni et al. [13], the environment is cluttered but a goal direction is not given. In Baldassarre et al. [14], both elements can be present at the same time but the synthesized solution, rather than exploiting direct local communication, uses instead indirect communication via specialized hardware. In this paper, we propose a novel negotiation strategy for collective transport in presence of both obstacles and of a goal. The proposed negotiation strategy is based on local direct communication.

The remaining of the paper is organized as follows. In Section 2 we describe the task and the simulated robots. In Section 3 , we describe the method we propose to design the controller. In Section 4, we present experimental results, whereas in Section 5 we conclude and sketch possible future works.

\section{Task Definition}

A group of three identical simulated mobile robots (like the one depicted in Figure 1 a attach to an irregularly shaped object(b) The task is to collectively transport the object from an initial to a goal location. The robots we used are modeled after the foot-bot robot [15], developed within the Swarmanoid project 1 . The irregularly shaped object is an object which cannot be grasped through its entire perimeter but only in certain regions. In our case, it is another simulated robot of the Swarmanoid project, the hand-bot (Figure 1b] [16]. This robot is a manipulator that does not have locomotion capabilities and thus needs to be carried by the foot-bots. In this task, the hand-bot is passive during the entire process.

1 http: / / www. swarmanoid.org 
The environment is an arena where a number of cuboid-shaped obstacles are present, each with an arbitrary position and orientation. Each of the three simulated mobile robots is equipped with a number of sensors and actuators. We considered and used only the following sensors and actuators: i) a light sensor, that is able to perceive the intensity of the light coming from different directions around the robot; ii) a distance scanner, that is used to obtain distance and angular values from the robot to other objects in the environment [17]; iii) a range and bearing communication system, with which a robot can send a message to other nearby robots in line of sight [18]; iv) a gripper, that is used to physically connect to the transported robot considered in the experiment; v) a turret actuator which, when set to active mode, can be used to rotate the gripper installed on a rotating ring or, when set to passive mode, can freely rotate in accordance with the speed of the wheels when the gripper is gripping an heavy object; vi) a wheels actuator, that is used to control independently the speed of the left and right wheels of the robot. The light sensor and the distance scanner sensor are not perfect but subject to a certain degree of noise. The range and bearing communication device can perceive messages coming from up to 4 meters away, more than enough to guarantee communication between the robots when connected. The distance scanner has a range of 1.5 meters.

In the experiments, we also place a light source in a fixed position in the environment behind the goal area. The light source has a high intensity such that it can be perceived by all the robots. The aim of the light source is to act as a common environmental cue, which is used as an implicit and shared reference frame by the robots.

For the sake of simplicity, the robots use the direction of the light source as the goal direction, that is they perform photo-taxis. Since the proposed methodology is not restricted to this case, in Section 3 we consider the goal direction and the environmental cue (or light) direction as two separated concepts. In the case where the goal direction is different from the light direction, the robot might need to be equipped with a separate sensor to detect the goal direction.

The presence of obstacles and the need to move to a given goal location create the need of handling conflicting individual decisions, which can be produced due to the non uniform perception of the environment.

For each individual robot, information of the following nature can be available at a given time:

No information: The goal is not perceived, for example because occluded by obstacles, and no obstacles are perceived as well.

Goal only: Only the goal is perceived, hence the robot moves towards it.

Obstacle only: The robot does not perceive the goal. However, it perceives an obstacle, hence it has to avoid it. At the same time, it has to inform other robots about the obstacle avoidance direction.

Goal and obstacle: The robot perceives both the goal and an obstacle. The direction of movement, considered by the robot and communicated to the other robots, has to take into account both these elements. 
Table 1 Explanation of the notation used to describe the two behaviors

\begin{tabular}{|c|l|l|}
\hline Notation & Meaning & Behavior \\
\hline \hline$\theta_{P}$ & Preferred direction when in $S_{\text {stubborn }}$ state & Social mediation \\
\hline$\theta_{S}$ & Socially mediated angle $\theta_{S} \leftarrow \angle \sum_{i=0}^{k} e^{j \theta_{i}}$ & $\begin{array}{l}\text { Social mediation, collective } \\
\text { transport }\end{array}$ \\
\hline$\theta_{0}$ & $\begin{array}{l}\text { Direction sent by social mediation behavior: } \theta_{S} \text { in } \\
S_{\text {Social }} \text { state or } \theta_{P} \text { in } S_{\text {stubborn }} \text { state }\end{array}$ & Social mediation \\
\hline$\theta_{1} \ldots \theta_{k}$ & Direction received from the $k$ neighbors & Social mediation \\
\hline$\theta_{G}$ & Goal direction & Collective transport \\
\hline$\theta_{C O}$ & Obstacle direction & Collective transport \\
\hline$\theta_{O A}$ & $\begin{array}{l}\text { Obstacle avoidance direction. It has to take into ac- } \\
\text { count also } \theta_{G} \text { if the goal is perceived. }\end{array}$ & Collective transport \\
\hline$\theta_{F}$ & $\begin{array}{l}\text { Direction of the shared environmental cue. All } \\
\text { other directions are always relative to this }\end{array}$ & Collective transport \\
\hline $\bar{\theta}_{S}$ & Weighted time average of $\theta_{S}$ & Collective transport \\
\hline
\end{tabular}

We now have all the elements to introduce the method we propose for tackling this task.

\section{Method}

In this section we first introduce the main idea behind the proposed method. Subsequently, we present the collective transport behavior, which we decomposed into three sub-behaviors: go to goal, obstacle avoidance and social mediation.

In the following, we will use a certain notation to denote directional information used in the behaviors. This is explained and summarized in Table 1

The low level behaviors go to goal and obstacle avoidance are used as follows. The go to goal behavior is used to query sensors and to obtain a goal direction, denoted as $\theta_{G}$; the obstacle avoidance behavior is used to detect the presence of obstacles and the angle $\theta_{C O}$ of the closest one. The social mediation and collective transport behaviors are the core focus of the proposed method.

The social mediation behavior, explained in Section 3.1 is used to negotiate the direction to be followed in collective transport. This is needed since, as explained in Section 2, different robots in the group can have access to conflicting information, for example one might perceive the goal as well as an obstacle while the others might perceive just the goal. Furthermore, when two or more robots perceive an obstacle, they can perceive it from different angles.

Once a collective decision has been made on the direction to be followed, this is used by the collective transport behavior, explained more in details in Section 3.2 .

\subsection{Social Mediation}

The social mediation behavior is responsible for the negotiation of the direction of motion. The behavior uses the directional information given by $\theta_{S}$ and $\theta_{P}: \theta_{S}$ 
represents a socially mediated heading direction and $\theta_{P}$ the robot desired heading direction. The main idea behind the algorithm is the following. When a robot in the group has no information (i.e., it does not have any information on the goal or on the obstacles), it has an internal state set to $S_{\text {social }}$. In this state, the robot acts as a repeater, that is, it computes $\theta_{S}$, the average of the direction information available to its neighbors, and it sends this value around. However, when information (such as on the obstacle) is available to the robot, its internal state is set to $S_{\text {stubborn }}$. In this state, it will relay its own preferred direction $\theta_{P}$ (for example the obstacle avoidance direction) instead of $\theta_{S}$. When all other robots are still sending $\theta_{S}$, the opinion of the stubborn robot will soon diffuse in the entire group, that is $\theta_{S}$ through the group will converge to $\theta_{P}$. The internal state of this behavior can be changed only by the overall collective transport behavior, as explained in Section 3.2. Algorithm 1 depicts the steps executed at every control step.

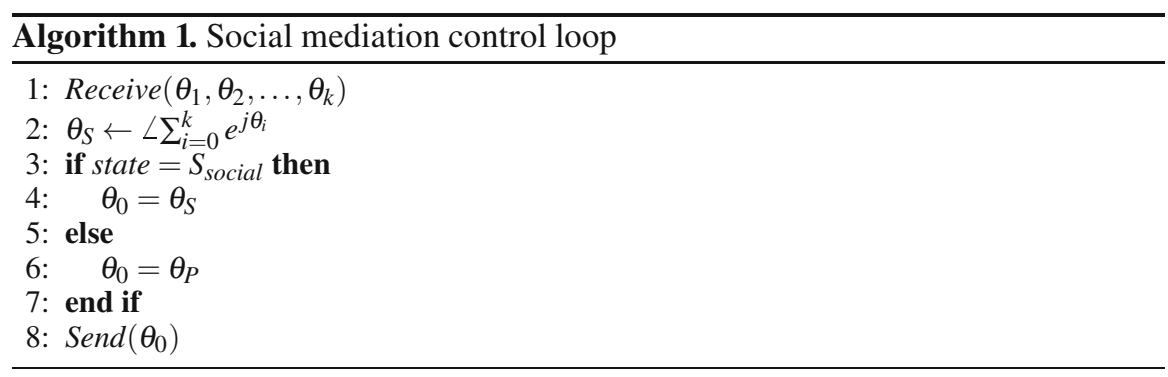

At the beginning of the control loop, the robot receives the heading direction information $\theta_{1}, \theta_{2}, \ldots, \theta_{k}$ of its neighbors, where $k$ is the number of neighbors. Communication is restricted to all neighboring robots in line of sight [18], as we are using the range and bearing communication mechanism. Due to this restriction, the

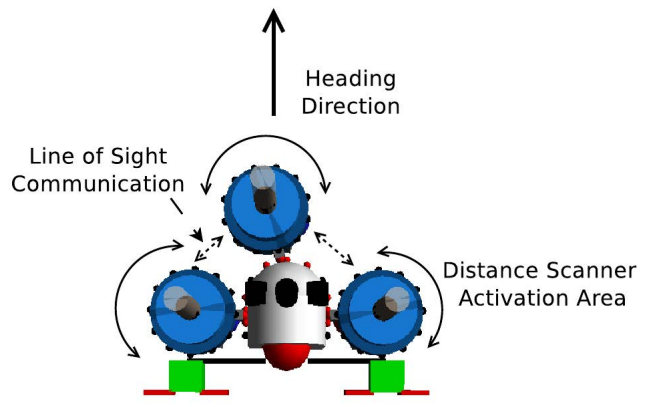

Fig. 2 The carried robot and the carrying robots. The circular arrows show the area of the distance scanner which is active for sensing, whereas dashed straight arrow show the line of sight communication relationships. 
robot attached at the center has $k=2$ neighbors, whereas the other two have $k=1$ neighbor (see Figure 2). The socially mediated heading $\theta_{S}$ is computed by averaging the directional information ( $\angle$ means "the angle of") received by the neighbors (line $2)$, with the robot's own information $\theta_{0}$.

By using the mechanism depicted above, we are solving the issue of how to diffuse a heading direction information, perceived only by one robot, through the entire group, without the need of special signaling. This allows all robots in a group to be aware of the avoidance direction of an obstacle, even if only one member of the group can perceive the obstacle.

In the following section, we describe how this mechanism is used to achieve effective collective transport with obstacle avoidance.

\subsection{Collective Transport and Obstacle Avoidance}

In this section we present the behavior responsible for collective transport with obstacle avoidance. This behavior uses the directional information computed in the social mediation behavior. In this behavior, $\theta_{O}$ denotes the direction of the obstacle (if perceived), $\theta_{G}$ denotes the goal direction (if the goal is perceived) and $\theta_{O A}$ denotes the obstacle avoidance direction (see table 1 for a summary). This directional information is always considered as relative to the direction of the shared environmental cue, denoted with $\theta_{F}$ and represented in our case by the light source.

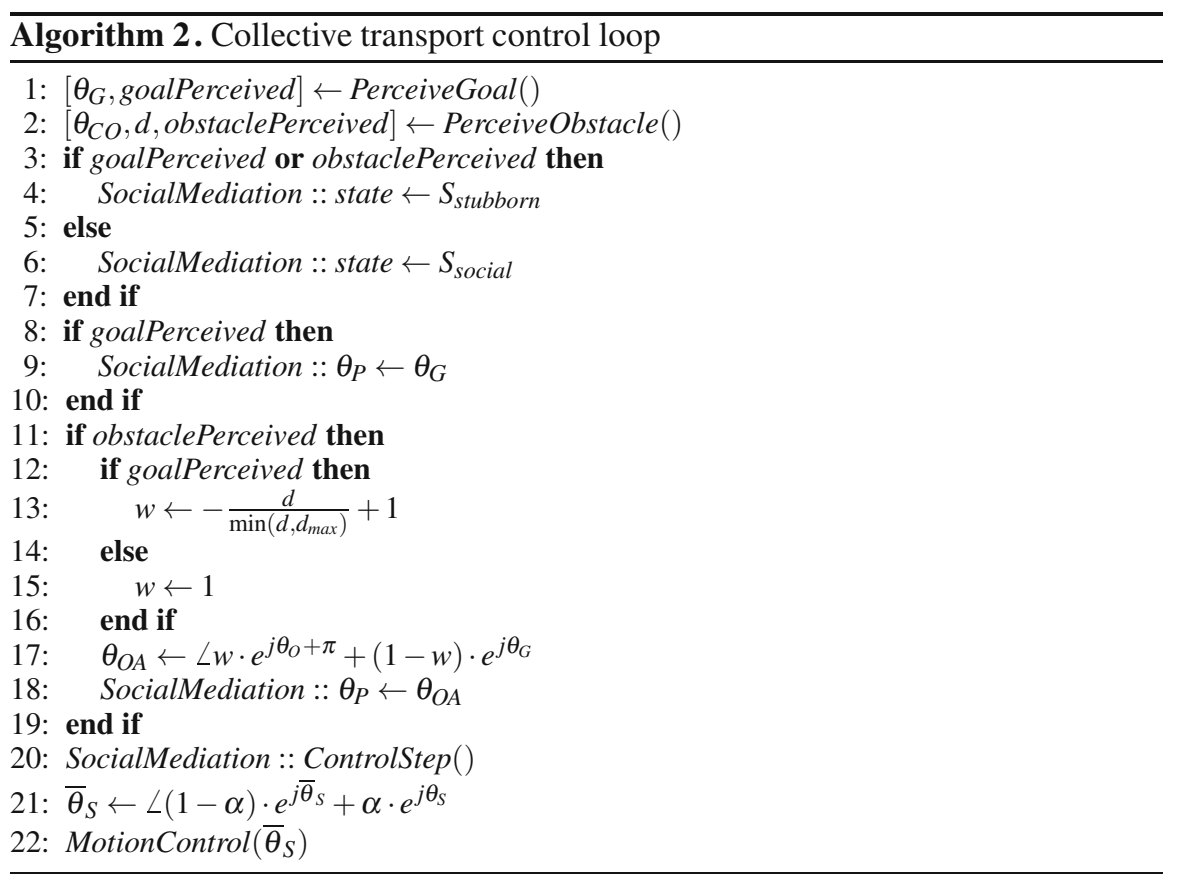


At the beginning of Algorithm 2, sensors are queried to detect whether the goal and/or obstacles are perceived (lines 1-2). The corresponding directions $\theta_{G}$, corresponding to the goal direction, and $\theta_{C O}$, corresponding to the angle of the closest obstacle, are also queried.

According to the information available to the robot (see Section 2) the internal state of the social mediation behavior is set (lines 3-7). If the robot perceives an obstacle with its distance scanner its state is set to $S_{\text {stubborn }}$. The same happens when the robot perceives the goal. In all other cases, that is when both the goal and the obstacles are not perceived, the state is set to $S_{\text {social }}$.

If the goal is perceived, the robot simply informs the others about the goal by setting its desired direction $\theta_{P}$ to the goal direction $\theta_{G}$ (line 9).

In case an obstacle is perceived two things can happen. If no goal direction $\theta_{G}$ is available, the robot simply tries to avoid the obstacle using the angle $\theta_{O A}=\theta_{C O}+$ $\pi$ and by setting $w=1$ (line 15). If, however, both the obstacle and the goal are perceived, the robot needs to compute the desired direction according to this two pieces of information: $\theta_{O}$ and $\theta_{G}$ are thus averaged using a weighted average and the result is assigned to $\theta_{O A}$ (lines 17). The weighted average uses a weight $w \in$ $[0,1]$ dependent on the distance between the robot and the obstacle (line 13) which represents how urgent it is to avoid obstacles: it is 1 when the obstacle is very close $(d=0)$ and 0 when it is far away $\left(d=d_{\max }\right.$, the maximal perception range of the obstacle avoidance behavior). We set $d_{\max }=0.75$ meters, half of the maximal range of the distance scanner, and we use the min operator to avoid negative values for $w$. The angle $\theta_{O A}$ is then assigned to the desired direction $\theta_{P}$ of the social mediation behavior (line 18).

Once $\theta_{P}$ is computed, the control step of the social mediation behavior is executed (line 20). As a result, the angle $\theta_{S}$ is computed by the social mediation behavior. This angle is then filtered by computing a time average (line 21) to filter out the effect of noise.

Finally, the motion control logic uses the filtered socially mediated direction $\bar{\theta}_{S}$ as a reference direction to be followed. The robot first converts the socially mediated direction to its local frame of reference using the common environmental cue direction $\theta_{F}$. All robots then compute the left and right wheels speed in the following way:

$$
N_{L}=u+\omega b, N_{R}=u-\omega b, \omega=K_{p} \bar{\theta}_{S},
$$

where $N_{L}, N_{R}$ are the wheels rotation speed of the left/right wheel speed respectively, $b$ is the distance between the center of the robot and each of the wheels, $u$ and $\omega$ are the forward and angular velocities respectively. The forward velocity $u$ is kept constant, whereas we vary the angular velocity $\omega$ proportionally to the socially mediated direction $\bar{\theta}_{S}$ to be followed, where $K_{p}$ is a proportional factor (we assume a clockwise convention for the angles). Furthermore, the motion control rule considers the robot attached to the left as the left wheel of the compound system and the robot attached to the right as the right wheel. This assumes that the two robots have always the direction of the wheels axis parallel to each other and it is ensured by the fact that we set the turret to active mode. Hence, the robot attached to the left of the 
compound will set both wheels speed to $N_{L}$, whereas the robot to the right will set them to $N_{R}$. The robot at the center can instead independently control its own left and right wheels depending on value computed by the motion control logic. The turret of the central robot, which is set to passive mode, freely rotates passively and follows the dynamics of the compound and the one imposed by the wheels.

To summarize the idea, the collective transport behavior interacts with the social mediation behavior to obtain a socially mediated direction $\bar{\theta}_{S}$ which is consistent in the group and allows a coherent motion. The social mediation behavior needs to be set in the appropriate state $\left(S_{\text {stubborn }}\right.$ or $\left.S_{\text {social }}\right)$, according to which information is available to the robot. It also needs the direction $\theta_{P}$ to be sent to the neighbors in case it is in $S_{\text {stubborn }}$ state. $\theta_{P}$ can be the direction to the goal, the obstacle avoidance direction or the direction which takes into account both the goal and the obstacles. The behavior achieves coherent collective motion even in case of conflicting opinions, since the motion control logic uses the socially mediated direction, that is the direction negotiated through the entire group, as the target direction to be followed.

\section{Experiments and Results}

We performed three sets of experiments. The first two sets consider a simple environment, where we position an obstacle at the center of the arena with varying angle $\alpha$ (see Figure 3a). For each setting, we executed 100 runs. Our prior expectation is that the more $\alpha$ tends to 0 , the longer it takes to avoid the obstacle in collective transport. We also expect that the proposed behavior is robust enough to always accomplish the task (move from an initial to a goal location, see Figure $3 \mathrm{~b}$ ) in this simplified setting. We hence report the completion times as a function of $\alpha$. The difference between the first and the second set of experiments is that in the first set we just analyze the impact of the angle $\alpha$ by keeping the projected size of the obstacle $m$ fixed (Figure 3a), whereas in the second set we also analyze the impact of the varying projected size, keeping $l$ fixed. Execution times are reported in time-steps. Each simulated second corresponds to 10 time-steps.

In the third and last set of experiments, we generate at random some more complex environments, of the type depicted in Figure $3 \mathrm{~b}$. We report the success rate of the behavior. We executed a total of 1000 runs, where in each run the angle and an offset of the position of each obstacle is generated at random.

Figure 4 shows the results for the first two sets of experiments performed in the simple environments. As we can see, the initial hypothesis can be accepted, as the execution times solely depends on $\alpha$ and not on the projected length $m$ of the obstacle. In fact, execution times increase with increasing values for $\alpha$. The more the obstacle is perpendicular to the direction of motion, the longer it takes for the robots to to perform obstacle avoidance.

The case $\alpha=0$ is particularly problematic. Average times are much higher, and many more outliers are present (not fully shown due to scale differences). This is explained by the fact that, when the obstacle is perpendicular to the direction of 


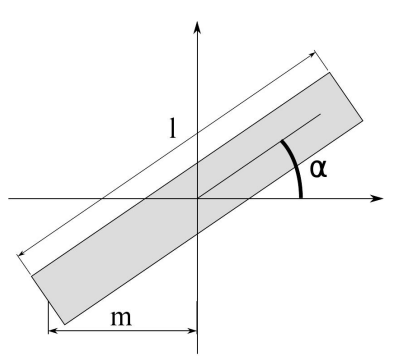

(a)

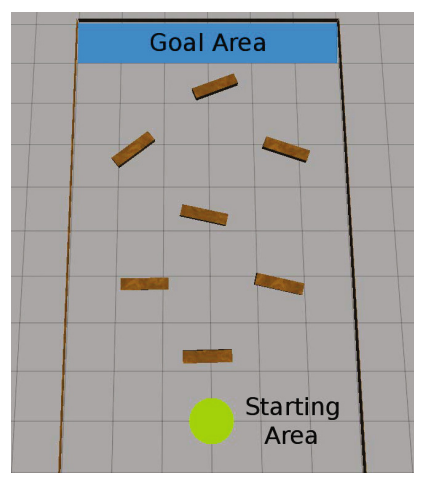

(b)

Fig. 3 (a) The controlled obstacle's parameter in the first two sets of experiments and (b) an example of complex environment. S denotes the starting area, $\mathrm{G}$ the goal area.

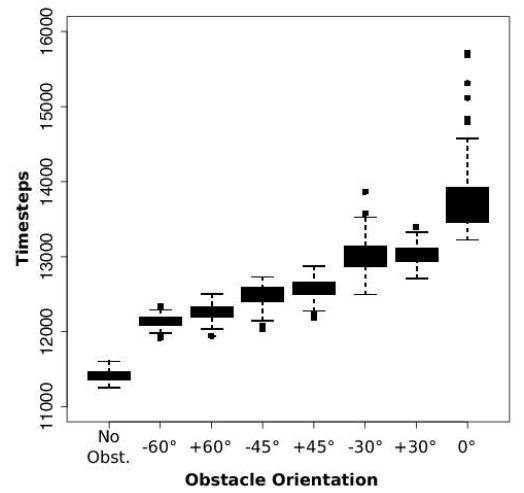

(a)

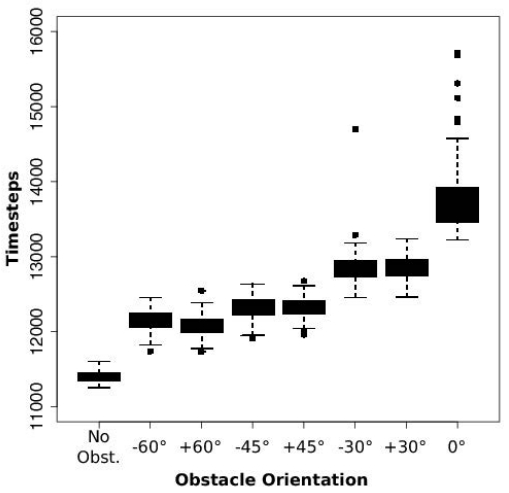

(b)

Fig. 4 Box plot of completion time for the experiment set with fixed $m$ (a) and for fixed $l$ (b)

motion, i.e. $\alpha=0$, the avoidance direction $\theta_{O A}$ takes some time to converge to one of the two possible obstacle avoidance sides. All the runs were successful and no collision was registered.

In the third set of experiments, results showed a remarkable success rate of $96 \%$. In the remaining $4 \%$ of the cases, robots hit an obstacle and hence the corresponding run was terminated. After analyzing failures cases separately, we found out that they were all due to slow turning rate achieved by the compound robot structure in the goal direction after avoiding an obstacle. This slow turning rate made the robot hit the next obstacle with the blind side of the carried structure, corresponding to the region of the object where the robots cannot attach and which is blind with respect 
to the distance scanner. A video showing one typical run for this set of experiments can be found in a supplementary page [19].

\section{Conclusion and Future Work}

In this paper, we presented a novel method to tackle a task that has received limited attention in the literature: obstacle avoidance in collective transport. The task involves collective transport of an object by a group of three robots. In this task, robots assemble to the object and have to navigate to a given goal location while avoiding obstacles.

The proposed method consists of two interacting behaviors. The first behavior is called social mediation and is used to perform negotiation of an heading direction which takes into account possibly conflicting perceptions of the members of the group. The second behavior achieves collective transport, using this mediated heading direction.

Experiments were performed in a simple arena with one obstacle placed at different angles and in a more complex arena with several obstacles. Results in the simple arena show that the efficiency (inversely linked to execution times) of the behavior solely depends on the angle at which obstacles are placed, and that the more the obstacle is placed perpendicularly to the direction of motion the more time it takes to avoid it. In a more complex environment, we measured the success rate of the proposed approach, obtaining $96 \%$ of success.

This work can be extended in a number of directions. As a first step, the proposed methodology can be validated on real robots. We speculate that the social mediation method, being a very high level behavior, will need few adaptations for the real robots experiments, whereas the collective transport might need some adjustments, especially for the motion control rule that has to minimize wheel slippage. Second, some of the assumptions made in this work could be relaxed. For example, it can be interesting to investigate how to solve the task by assuming that the irregular shape of the object is not known in advance. In this case, we speculate that the motion control logic will need to be extended. Third and more ambitiously, a long term goal would be to understand how to control a group of an arbitrary number of robots, connected between each other and/or to an irregular object at different positions. In this case, we speculate that the social mediation methodology can be extended to tackle dynamic negotiation of heading direction with an arbitrary number of robots. Finally, a theoretical model of the system can be developed and used to prove some properties of the algorithm, such as that no cyclic situations (i.e. no "deadlocks") can arise.

Acknowledgements. This work was supported by the SWARMANOID project funded by the Future and Emerging Technologies programme (IST-FET) of the European Commission (grant IST-022888). This work was partially supported by the European Union through the ERC Advance Grant "E-SWARM: Engineering Swarm Intelligence Systems" (contract 
246939). M. Birattari and M. Dorigo acknowledge support from the F.R.S.-FNRS of the French Community of Belgium.

\section{References}

1. Bahçeci, E., Soysal, O., Şahin, E.: A review: pattern formation and adaptation in multirobot systems. Technical Report CMU-RI-TR-03-43, Robotics Institute, Pittsburgh, PA (2003)

2. Varghese, B., McKee, G.: A review and implementation of swarm pattern formation and transformation models. International Journal of Intelligent Computing and Cybernetics 2(4), 786-817 (2009)

3. Balch, T., Hybinette, M.: Social potentials for scalable multi-robot formations. In: Carlisle, B.R., Khatib, O. (eds.) Proceedings of the 2000 IEEE International Conference on Robotics and Automation (ICRA 2000), pp. 73-80. IEEE Press, Piscataway (2000)

4. Spears, W.M., Spears, D.F., Hamann, J.C., Heil, R.: Distributed, physics-based control of swarms of vehicles. Autonomous Robots 17(2-3), 137-162 (2004)

5. Stilwell, D., Bay, J.: Toward the development of a material transport system using swarms of ant-like robots. In: Proceedings IEEE International Conference on Robotics and Automation, pp. 766-771. IEEE Comput. Soc. Press, Piscataway (1993)

6. Kosuge, K., Oosumi, T., Satou, M., Chiba, K., Takeo, K.: Transportation of a single object by two decentralized-controlled nonholonomic mobile robots. In: Giralt, G., Dario, P. (eds.) IEEE International Conference on Robotics and Automation, vol. 4, pp. 29892994. IEEE Press, Piscataway (1998)

7. Wang, Z., Takano, Y., Hirata, Y., Kosuge, K.: A pushing leader based decentralized control method for cooperative object transportation. In: Asama, H. (ed.) IEEE/RSJ International Conference on Intelligent Robots and Systems, vol. 1, pp. 1035-1040. IEEE Press, Piscataway (2004)

8. Balch, T., Ronald, Arkin, R.C.: Communication in reactive multiagent robotic systems. Autonomous Robots 1, 27-52 (1994)

9. Donald, B.R., Jennings, J., Rus, D.: Information invariants for distributed manipulation. The International Journal of Robotics Research 16(5), 673 (1997)

10. Yamada, S., Saito, J.: Adaptive action selection without explicit communication for multi-robot box-pushing. In: Harashima, F., Fukuda, T. (eds.) IEEE/RSJ International Conference on Intelligent Robots and Systems, vol. 3, pp. 1444-1449. IEEE Press, Piscataway (1999)

11. Campo, A., Nouyan, S., Birattari, M., Groß, R., Dorigo, M.: Negotiation of Goal Direction for Cooperative Transport. In: Dorigo, M., Gambardella, L.M., Birattari, M., Martinoli, A., Poli, R., Stützle, T. (eds.) ANTS 2006. LNCS, vol. 4150, pp. 191-202. Springer, Heidelberg (2006)

12. Groß, R., Dorigo, M.: Towards group transport by swarms of robots. International Journal of Bio-Inspired Computation 1(1-2), 1-13 (2009)

13. Trianni, V., Dorigo, M.: Self-organisation and communication in groups of simulated and physical robots. Biological Cybernetics 95, 213-231 (2006)

14. Baldassarre, G., Parisi, D., Nolfi, S.: Distributed coordination of simulated robots based on self-organization. Artificial Life 12(3), 289-743 (2006)

15. Bonani, M., Longchamp, V., Magnenat, S., Rétornaz, P., Burnier, D., Roulet, G., Vaussard, F., Bleuler, H., Mondada, F.: The MarXbot, a miniature mobile robot opening new perspectives for the collective-robotic research. In: Huo, R., Asama, H. (eds.) 2010 IEEE/RSJ International Conference on Intelligent Robots and Systems (IROS 2010). IEEE Press, Piscataway (2010) 
16. Bonani, M., Magnenat, S., Rétornaz, P., Mondada, F.: The Hand-Bot, a Robot Design for Simultaneous Climbing and Manipulation. In: Xie, M., Xiong, Y., Xiong, C., Liu, H., Hu, Z. (eds.) ICIRA 2009. LNCS (LNAI), vol. 5928, pp. 11-22. Springer, Heidelberg (2009)

17. Magnenat, S., Longchamp, V., Bonani, M., Rétornaz, P., Germano, P., Bleuler, H., Mondada, F.: Affordable slam through the co-design of hardware and methodology. In: Snyder, W., Kumar, V. (eds.) IEEE International Conference on Robotics and Automation. IEEE Press, Piscataway (2010)

18. Roberts, J., Stirling, T., Zufferey, J., Floreano, D.: $2.5 \mathrm{~d}$ infrared range and bearing system for collective robotics. In: Hamel, W.R. (ed.) IEEE/RSJ International Conference on Intelligent Robots and Systems. IEEE Press, Piscataway (2009)

19. Ferrante, E., Brambilla, M., Birattari, M., Dorigo, M.: "Look-out!": Socially mediated obstacle avoidance in collective transport: Complete data (2010), Supplementary information page at http://iridia.ulb.ac.be/supp/IridiaSupp2010-005/ 